\title{
Does Information About What Things Are Influence Children's Memory for Where Things Are?
}

\author{
Alycia M. Hund and Jodie M. Plumert \\ University of Iowa
}

\begin{abstract}
Two experiments examined how information about what objects are influences memory for where objects are located. Seven-, 9-, and 11-year-old children and adults learned the locations of 20 objects marked by dots on the floor of a box. The objects belonged to 4 categories. In one condition, objects belonging to the same category were located in the same quadrant of the box. In another condition, objects and locations were randomly paired. After learning, participants attempted to replace the objects without the aid of the dots. Children and adults placed the objects in the same quadrant closer together when they were related than when they were unrelated, indicating that object information led to systematic biases in location memory.
\end{abstract}

Remembering where things are is central to human functioning. Children and adults must be able to remember locations to carry out basic tasks such as getting to school or preparing a snack. For the vast majority of such tasks, people must remember the links between objects and locations. For example, knowing that bread, peanut butter, and jelly are needed to make a sandwich is not very useful if one does not know where these things are located in the kitchen. In fact, not remembering the links between objects and their locations can lead to long and tedious searches. Despite the importance of linking objects with locations, research on memory for location has traditionally focused on how children and adults exploit spatial sources of information to remember locations. For example, many studies have examined how children and adults use boundaries and landmarks to remember locations (e.g., Acredolo \& Evans, 1980; Holyoak \& Mah, 1982; Kosslyn, Pick, \& Fariello, 1974; McNamara, 1986; McNamara \& Diwadkar, 1997; Newcombe \& Liben, 1982; Sadalla, Burroughs, \& Staplin, 1980). Very little is known about how people use nonspatial sources of information to remember the locations of objects. Our goal in the present investigation was to examine how information about objects influences memory for locations.

How might children and adults remember object locations? Recent work suggests that people use two sources of information to estimate location - fine-grained information about the location

Alycia M. Hund and Jodie M. Plumert, Department of Psychology, University of Iowa.

This research was supported by National Institutes of Health Grant R03-HD36761-01 awarded to Jodie M. Plumert. We thank Royelle Hoffman, Peter Lovegrove, and Kim Schroeder for their help with data collection and coding. We also thank the Regina Elementary School in Iowa City, Iowa, and the College Community Schools in Cedar Rapids, Iowa, for their cooperation.

Correspondence concerning this article should be addressed to Alycia M. Hund, who is now at the Department of Psychology, Illinois State University, Campus Box 4620, Normal, Illinois 61790-4620, or to Jodie M. Plumert, Department of Psychology, 11 Seashore Hall East, University of Iowa, Iowa City, Iowa 52242. E-mail: amhund@ilstu.edu or jodie-plumert @uiowa.edu to be remembered and coarse-grained information about the category to which the location belongs (e.g., Engebretson \& Huttenlocher, 1996; Hund \& Plumert, 2002; Hund, Plumert, \& Benney, 2002; Huttenlocher, Hedges, \& Duncan, 1991; Huttenlocher, Newcombe, \& Sandberg, 1994; Plumert \& Hund, 2001; Sandberg, Huttenlocher, \& Newcombe, 1996). These ideas have been formalized in the category adjustment (CA) model of location estimation (Huttenlocher et al., 1991). When trying to remember a previously learned location, people make estimates based on their memory of fine-grained metric information, such as distance and direction from an edge. However, because memory for finegrained information is inexact, people adjust these estimates on the basis of categorical information about the location (i.e., region membership). According to the model, this categorical information is represented by a prototype located at the center of the spatial region. Hence, adjustments based on spatial category information lead to systematic distortions toward the centers of spatial categories. In addition, the magnitude of distortion depends on the certainty of fine-grained metric information. When memory for fine-grained information is relatively certain, people assign a low weight to categorical information, which results in minimal distortions toward the category centers. Conversely, when memory for fine-grained information is uncertain, people assign a high weight to categorical information, which results in relatively large distortions toward the category centers.

Clearly, spatial categories play an important role in people's memory for object locations. To date, most of the research based on the CA model has defined spatial categories as geometric regions of space delineated by visible or mentally imposed boundaries (e.g., Huttenlocher et al., 1991, 1994; Plumert \& Hund, 2001). For example, children and adults use the edges of a rectangular sandbox to delineate the category "in the sandbox." Moreover, older children and adults use the midline symmetry axis of the sandbox to further subdivide it into two spatial categories: the right half and the left half (Huttenlocher et al., 1994). This general proposal that children and adults use geometric regions as spatial categories has received considerable support (e.g., Engebretson \& Huttenlocher, 1996; Hund \& Plumert, 2002; Hund \& Spencer, 
2003; Huttenlocher et al., 1991; Plumert \& Hund, 2001; Sandberg et al., 1996; Spencer \& Hund, 2002, in press).

One problem with relying only on geometric cues to define spatial categories is that not all spaces can be easily divided into geometric regions on the basis of symmetry axes or visible boundaries. Some spaces are irregularly shaped, making it difficult to impose symmetry axes. Other spaces may lack salient boundaries, making it difficult to rely on such cues to subdivide the space. Given these problems, people may need to rely on other types of cues to delineate spatial categories. For example, people might use spatiotemporal experience to form spatial categories. In other words, people might group together a set of locations on the basis of visiting those locations close together in time. Once such a category is formed, people could use information about the category to adjust their estimates of location. In this case, the categorical information would be represented by the center of the group of locations. Hence, adjustments based on spatial category information would lead to systematic distortions toward the centers of spatial groups (Hund et al., 2002). Support for this broader view of spatial categories comes from several sources (e.g., Clayton \& Habibi, 1991; Curiel \& Radvansky, 1998; Hund et al., 2002; McNamara, Halpin, \& Hardy, 1992; Sherman \& Lim, 1991).

Our goal in the present investigation was to examine how one nongeometric cue-object information-influences categorical bias in estimates of location. More specifically, do children and adults remember related objects as closer together than they really are? Why might object relatedness influence memory for location? Quite simply, related objects often occupy nearby locations. For example, toys belonging to the same thematic categories are often located together in children's preschool rooms: food and dishes in the kitchen area; puzzles, games, and books in the study area; and paints, smocks, and easels in the art area. In fact, even toddlers are sensitive to the confluence of object function cues and spatial cues (e.g., Mandler \& Bauer, 1988; Mandler, Fivush, \& Reznick, 1987). That is, 20-month-olds recognize contextual categories (e.g., kitchen things) whose members share similar functions and locations. These findings suggest that everyday experience with related objects occupying nearby locations might lead children and adults to use information about what things are to help them remember where things are.

Although research investigating how object information influences memory for location is scarce, previous findings suggest that nonspatial information can play an important role in the way people process spatial information. For example, people use object information to determine linguistic spatial relations (CarlsonRadvansky, Covey, \& Lattanzi, 1999; Carlson-Radvansky \& Tang, 2000). Carlson-Radvansky et al. (1999) asked adults to place a photograph of one object (e.g., a tube of toothpaste) above a photograph of a functionally related object (e.g., a toothbrush). Rather than placing the object above the center of mass of the base object, as predicted by theories in which what and where information do not interact, adults made placements that were biased toward the functional portion of the base object (e.g., the toothbrush bristles), suggesting that what an object is influences how people think about where it is.

Hirtle and colleagues (Hirtle \& Kallman, 1988; Hirtle \& Mascolo, 1986) also examined how object information might influence people's judgments about locations. Adults learned the locations of 10 items on a fictitious map. Five of the items belonged to the governmental cluster (e.g., city hall, police station), whereas five belonged to the recreation cluster (e.g., playground, beach). Following learning, participants judged the distance between pairs of items. Adults tended to underestimate the distance between items in the same cluster relative to equidistant items in different clusters. That is, adults recalled the police station and city hall as being closer together than the police station and the playground. These findings suggest that similarity among the items influenced memory for their locations (but see McNamara \& LeSueur, 1989).

Do children use nonspatial information to process spatial information? Recently, Uttal, Gregg, Tan, Chamberlin, and Sines (2001) examined how object information influences preschoolers' ability to use maps. Three-, 4-, and 5-year-old children viewed a map depicting 27 locations organized as a schematic figure of a dog. For half of the children, lines on the map connected the locations, making the dog figure very salient. For the remaining children, no lines were present. Children in both conditions used markings on the map to find stickers hidden at the corresponding locations in a larger space. Seeing the lines did not influence the performance of the younger children. However, the 5-year-olds in the lines condition were significantly more accurate when searching for the stickers than were the 5-year-olds in the no-lines condition, which suggests that object information improved their ability to search correctly. These findings provide preliminary support for the notion that children (i.e., 5-year-olds) use nonspatial information to process spatial information.

The present investigation directly examined how 7- to 11-yearold children and adults use object information to remember locations. Specifically, we investigated whether 7-, 9-, and 11-year-old children and adults remember related objects as closer together than they really are. As in previous studies, children and adults learned the locations of 20 objects marked by yellow dots on the floor of an open, square box (Hund \& Plumert, 2002; Hund et al., 2002; Plumert \& Hund, 2001). The objects belonged to four categories: animals, vehicles, food, and clothing. In the related condition, objects belonging to the same object category were located in the same quadrant of the box. In the unrelated condition, the same objects and locations were used; however, they were randomly paired. Following learning, participants attempted to replace the objects in the correct locations without the aid of the dots marking the locations. We predicted that participants in the related condition would place the objects belonging to the same group closer together than would participants in the unrelated condition, which would suggest that children and adults use object information to facilitate memory for locations.

\section{Experiment 1}

\section{Method}

\section{Participants}

Ninety-six 7-, 9-, and 11-year-old children and adults participated. There were 24 participants in each age group, with approximately equal numbers of males and females in each group. The mean ages of the groups were 7 years 11 months (range $=7$ years 10 months to 8 years 1 month), 9 years 6 months (range $=9$ years 4 months to 9 years 10 months), 11 years 5 months (range $=10$ years 9 months to 12 years 1 month), and 19 years 10 months (range $=18$ years 4 months to 27 years 9 months). One additional 9-year-old was excluded because she did not reach our learning criterion. 
One additional 7-year-old was excluded because she misplaced 12 of 20 locations during the test phase. Children were recruited from a child research participant database maintained by the Department of Psychology at the University of Iowa. Parents received a letter describing the study followed by a telephone call inviting children to participate. Adults participated to fulfill research credit for an introductory psychology course.

\section{Apparatus and Materials}

A 32 -in. long $\times 32$-in. wide $\times 13$-in. high $(81.28 \mathrm{~cm} \times 81.28 \mathrm{~cm} \times$ $33.02 \mathrm{~cm}$ ) open, square box designed to look like a model house was used as the experimental space. The house had two identical windows evenly spaced on each of its four white exterior walls. The floor consisted of a layer of Plexiglas and a layer of plywood separated by a $1 / 2-$ in. $(1.27-\mathrm{cm})$ space. Removable boards could be inserted between the plywood and the Plexiglas to change the appearance of the floor. Three floors were used in this experiment: (a) a blue carpeted floor with yellow dots marking the locations, (b) a blue carpeted floor with no dots, and (c) a grid of $\mathrm{x}$ - and $\mathrm{y}$-coordinates at $1 / 2$-in. $(1.27-\mathrm{cm})$ intervals.

The dot floor contained 20 locations marked by 3/4-in. (1.91-cm) yellow dots (see Figure 1). Twenty miniature objects were used to help participants learn the locations on the floor: a car, a truck, a train, a van, a cement mixer, a shirt, a shoe, a hat, a skirt, a pair of gloves, an apple, a bag of chips, a pie, a soup can, a box of popcorn, a horse, a dog, a pig, a cow, and a cat. These objects belonged to four categories (i.e., vehicles, clothing, food, and animals). The average length and width of the objects were 0.97 in. $(2.46 \mathrm{~cm})$ and 0.61 in. $(1.55 \mathrm{~cm})$, respectively.

\section{Design and Procedure}

The model house was placed on the floor of the experimental room. The experimenter stood directly in front of the house, and participants were seated to the right of the experimenter facing an adjacent side of the house (see Figure 1).

Participants were randomly assigned to one of two experimental conditions: related or unrelated. In the related condition, the pairings of objects and locations were constrained so that all objects belonging to an object category occupied locations in the same quadrant of the model house. In the unrelated condition, the same objects and locations were used, but the

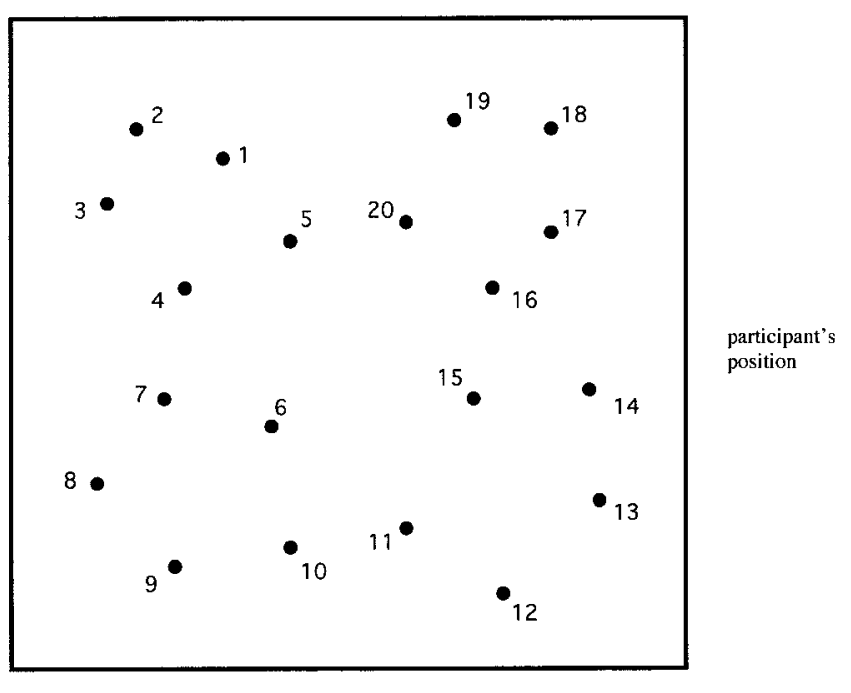

experimenter's position

Figure 1. Diagram of floor of model house and locations. Location numbers are for illustrative purposes only. pairings of objects and locations were completely randomized so that any object could occupy any location.

The experimental session included a learning phase followed by a test phase. During the learning phase, participants learned the locations of 20 objects in the house. At the beginning of the session, the experimenter told participants that 20 objects would be placed in the model house and that they should try to remember the locations of the objects because they would be asked to replace them later. The object locations corresponded to the 20 yellow dots on the floor of the house, including five in each quadrant (see Figure 1). Participants watched as the experimenter named the objects and placed them in the house one at a time in a random order. The pairings of locations and objects were randomized for each participant; however, these random pairings were constrained in the related condition such that objects belonging to the same object category were located in the same quadrant of the house. The pairings of object categories and spatial quadrants were also randomized for each participant in the related condition.

After the experimenter had placed all 20 objects, participants were asked to turn around while the experimenter removed the objects from the model house. The experimenter then gave the objects to the participants one at a time and asked them to place them in the model house. The participant's task was to try to place each object in its correct location. Incorrect placements were recorded and corrected by the experimenter. Participants were allowed to move around the outside of the model house to replace the objects during learning trials. The objects remained on the dots until all 20 had been placed. Participants received the objects in a new random temporal order for each learning trial. They continued with the learning trials until they could correctly replace all 20 objects (i.e., on the corresponding yellow dots) in a single trial. The mean numbers of trials to criterion for 7-, 9-, and 11-year-olds and adults were $3.42(S D=2.10), 3.67(S D=1.27)$, $3.29(S D=1.68)$, and $2.13(S D=0.95)$, respectively.

The test phase began immediately following the learning phase. First, the experimenter asked the participants to turn away from the model house while the objects were removed. The experimenter also removed the floor with the yellow dots and replaced it with a plain blue floor. Participants then were asked to face the house and try to replace the objects in the correct locations. Thus, participants attempted to place the objects in the correct locations without the aid of the yellow dots marking the locations. Participants replaced the objects in any order they chose.

After participants replaced the objects, the experimenter thanked them for participating. After participants left, the experimenter removed the blue floor, replaced it with the grid of $\mathrm{x}$ - and $\mathrm{y}$-coordinates, and recorded the $\mathrm{x}$ and $y$-coordinates for each object to the nearest $1 / 2$ in. $(1.27 \mathrm{~cm})$.

\section{Coding and Measures}

A placement was considered correct if it was in the correct quadrant of the model house and in the correct position relative to the other objects. Occasionally, participants preserved the overall configurations of locations but incorrectly paired objects and locations. As in other studies (e.g., Hund $\&$ Plumert, 2002; Hund et al., 2002; Plumert \& Hund, 2001), we used the $\mathrm{x}$ - and $\mathrm{y}$-coordinates for these locations regardless of whether the correct objects were placed in the locations. We substituted $3.54 \%$ of the locations for 7-year-olds (17 out of 480), 1.25\% for 9-year-olds (6 out of 480), $1.04 \%$ for 11 -year-olds ( 5 out of 480 ), and $0.83 \%$ for adults ( 4 out of 480 ). These substituted locations were used in all analyses. As in previous experiments, objects placed in the wrong quadrant or in a completely wrong configuration were omitted from analyses. We omitted $3.13 \%$ of locations for 7 -year-olds (15 out of 480 ), $4.17 \%$ for 9 -year-olds (20 out of 480 ), $1.25 \%$ for 11 -year-olds ( 6 out of 480 ), and $1.67 \%$ for adults ( 8 out of 480).

We calculated intercoder reliability estimates of object placement for 16 randomly selected participants ( $15 \%$ of the sample) using exact percentage agreement. For each of these participants, two coders judged which object was placed at each of the 20 locations. Coders agreed on $100 \%$ of the 320 locations coded. 
Mean error score. Participants received a single mean error score reflecting the degree to which they placed objects near their actual locations. This score was calculated by first determining the distance between each remembered and actual location and then averaging these distances over all locations to obtain a single mean error score that reflected the accuracy of memory for fine-grained information.

Variable error score. Participants received a single variable error score reflecting the variability of remembered placements relative to actual locations. This score was calculated by first determining the distance between each remembered and actual location and then calculating the standard deviation of these distances to obtain a single variable error score that reflected the precision of memory for fine-grained information.

Center displacement score. Participants also received a center displacement score reflecting the degree to which they systematically placed objects belonging to the same spatial group (i.e., in each quadrant) closer together than they actually were. To calculate this score, we first subtracted the distance between each remembered location and the center of mass of the remembered group of locations from the distance between the corresponding actual location and the center of mass of the actual group of locations. We then averaged these differences across all 20 locations to obtain a single center displacement score for each participant. Thus, the center displacement score reflected the degree to which participants displaced locations toward the centers of the spatial groups after we removed effects due to translation of groups. This score provided a sensitive index of categorical bias in estimates of location.

Spatial clustering score. Participants also received a spatial clustering score reflecting the degree to which they replaced objects quadrant by quadrant during test. The clustering measure used was the adjusted ratio of clustering (ARC) score (Roenker, Thompson, \& Brown, 1971). This score represents the proportion of the observed number of quadrant repetitions relative to the total possible number of repetitions corrected for chance. A score of 1.00 represents perfect clustering, whereas a score of 0.00 represents no above-chance clustering. ARC scores in this study ranged from 0.00 to 1.00 (negative ARC scores were set to 0 ). This score provided an index of spatial organization in participants' replacement orders during test.

\section{Results}

\section{Mean Error}

In general, children and adults placed the objects fairly accurately, which suggests that they used fine-grained metric information to estimate the locations. Mean error scores were entered into an Age ( 7 years vs. 9 years vs. 11 years vs. adult) $\times$ Condition (related vs. unrelated) analysis of variance (ANOVA). This yielded a significant main effect of age, $F(3,88)=11.15, p<.01$. Fisher's protected least significant difference (PLSD) follow-up tests indicated that the adults exhibited significantly less mean error than did the other age groups. In addition, the 11-year-olds exhibited significantly less error than did the 7- and 9-year-olds (see Table 1).

\section{Variable Error}

To investigate possible differences in the variability of placements among the age groups and experimental conditions, we entered variable error scores into an Age (4) $\times$ Condition (2) ANOVA. This yielded a significant main effect of age, $F(3,88)=$ $5.89, p<.01$. Fisher's PLSD follow-up tests indicated that the adults' placements were significantly less variable than were the 7and 9-year-olds' placements (see Table 1). In general, variability
Table 1

Mean and Variable Error Scores (in Centimeters) for Children and Adults in Experiment 1

\begin{tabular}{llc}
\hline Age group & Mean error & Variable error \\
\hline 7-year-olds & $5.97(1.04)$ & $2.97(.76)$ \\
9-year-olds & $5.77(.97)$ & $2.97(.51)$ \\
11-year-olds & $5.11(1.04)$ & $2.62(.64)$ \\
Adults & $4.37(1.17)$ & $2.31(.66)$ \\
\hline
\end{tabular}

Note. Standard deviations are listed in parentheses.

decreased across development, which suggests that the precision of fine-grained information increased across development.

\section{Center Displacement}

Did children and adults in the related condition place the objects closer together than they actually were? We used two sets of statistical analyses to address this question. One set examined differences in the magnitude of center displacement across ages and conditions. The second set of analyses examined whether the magnitude of center displacement was significantly greater than 0 for each age and condition. In the first analysis, we entered center displacement scores into an Age (4) $\times$ Condition (2) ANOVA to examine differences in displacement across age groups and conditions. As expected, this analysis yielded a significant main effect of condition, $F(1,88)=14.88, p<.01$. Participants in the related condition placed the objects significantly closer to the centers of the spatial categories $(M=1.09 \mathrm{~cm}, S D=1.30 \mathrm{~cm})$ than did participants in the unrelated condition $(M=0.15 \mathrm{~cm}, S D=1.19$ $\mathrm{cm})$.

In the second analysis, we conducted separate one-sample $t$ tests to determine whether the magnitude of displacement was significantly greater than 0 for participants in each age and condition. No difference in distance would be expected if participants neither underestimated nor overestimated the distances between locations belonging to each spatial category. Positive scores would reflect displacement toward the centers of the spatial categories, whereas negative scores would reflect displacement away from the centers of the categories. That is, if participants displaced objects toward the centers of the spatial categories, then the mean difference between the actual location to actual category center distance and the remembered location to remembered category center distance should be greater than 0 .

Seven- and 9-year-olds and adults in the related condition placed the objects significantly closer to the category centers than they actually were, all $t \mathrm{~s}(11)>3.13, p s<.01$. In contrast, 11 -year-olds in the related condition did not place the objects significantly closer to the category centers than they actually were, $t(11)=1.78, n s$. In the unrelated condition, 9-year-olds, 11-yearolds, and adults showed little categorical bias, all $t \mathrm{~s}(11)<1.72, n s$, and 7 -year-olds placed the objects significantly further from the category centers than they actually were, $t(11)=-2.69, p<.05$. Center displacement scores for the 7-, 9-, and 11-year-olds and the adults in each experimental condition are shown in Figure 2.

A center displacement score for each individual location was also calculated to determine whether the overall center displacement findings were driven by only a few locations. In particular, 


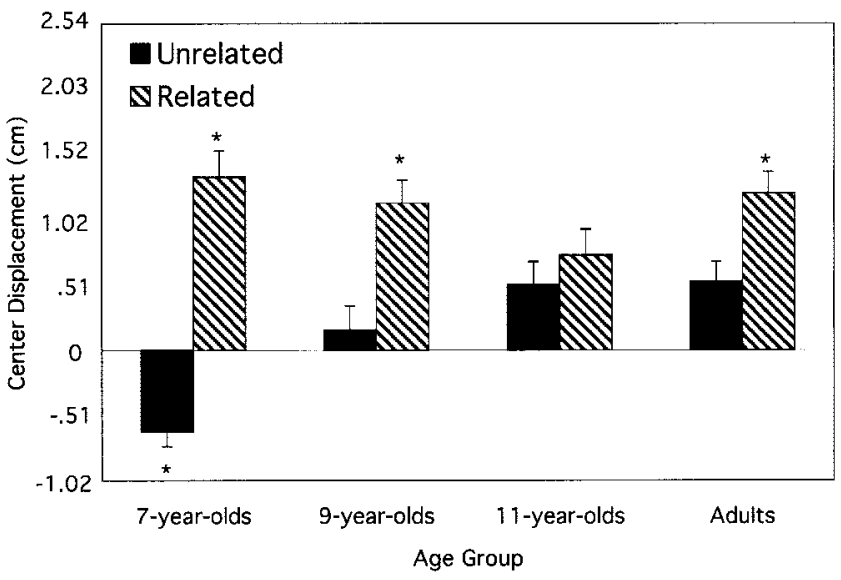

Figure 2. Center displacement scores for each age group and condition in Experiment 1. Asterisks denote significant results $(p<.05)$ of one-sample $t$ tests $(d f=11)$ comparing the displacement score with the expected score with no displacement (i.e., $0 \mathrm{~cm}$ ).

we explored whether the effects resulted from the "inner" locations (e.g., the locations near the center of the box) being placed further from each other than they really were. Center displacement scores for each location were compared with the expected score of 0 by using separate one-sample $t$ tests collapsed across age groups and conditions. Participants significantly displaced 13 of the locations (e.g., Locations 3, 5, 7, 8, 9, 10, 11, 12, 13, 14, 15, 16, and 19) toward the centers of the groups, all $t \mathrm{~s}(95)>2.30, p \mathrm{~s}<.05$. In addition, they displaced 3 of the locations (e.g., Locations 1, 17, and 20) away from the centers of the groups, all $t \mathrm{~s}(95)<-3.85$, $p s<.01$. The remaining 4 locations were not significantly displaced toward or away from the centers of the groups. Together, these results suggest that the overall displacement findings resulted from children and adults placing many of the locations closer to the centers of the groups than they really were. It is important to note that both inner locations (i.e., near the middle of the box) and outer locations (i.e., near the exterior walls of the box) were displaced toward the centers of the groups. Thus, the effects were not solely based on the inner locations being pushed away from each other.

\section{Spatial Clustering}

To investigate whether the order in which participants placed the objects during the test phase differed by age or condition, we entered spatial clustering (ARC) scores into an Age (4) $\times$ Condition (2) ANOVA. This analysis yielded significant main effects of age, $F(3,88)=5.14, p<.01$, and of condition, $F(1,88)=43.44$, $p<.01$. Fisher's PLSD follow-up tests indicated that the adults exhibited greater spatial organization in their placement orders during test $(M=.76, S D=.29)$ than did the other age groups (7-year-olds, $M=.48, S D=.35$; 9-year-olds, $M=.53, S D=.36$; 11-year-olds, $M=.65, S D=.31$ ). Similarly, the 11-year-olds exhibited greater spatial organization in their placement orders during test than did the 7-year-olds. Moreover, participants in the related condition were much more likely to place the objects belonging to one quadrant together in time during replacement $(M=.79, S D=.28)$ than were participants in the unrelated condition $(M=.42, S D=.29)$. ARC scores for each age group and condition can be seen in Table 2 .

\section{Discussion}

Our goal in this experiment was to examine how information about what objects are might influence memory for where objects are located. In other words, do children and adults remember related objects as closer together than they really are? In general, participants in the related condition placed the objects significantly closer to the centers of the spatial groups than did participants in the unrelated condition, indicating that they remembered related objects as closer together than unrelated objects. These findings demonstrate that information about what objects are influences children's memory for where objects are located.

Object information also influenced the order in which children and adults replaced the objects during test. Children and adults in the related condition tended to place the objects belonging to the same quadrant before placing the objects belonging to another quadrant. Moreover, adults exhibited more spatial organization in their replacement orders than did the children. Eleven-year-olds also exhibited more organization than did the 7-year-olds, which suggests that spatial organization during replacement increases across development. These results are consistent with other findings suggesting that spatial organizational strategies increase across childhood and adolescence (e.g., Plumert, 1994).

It is interesting that the present findings revealed that the magnitude of categorical bias followed a U-shaped developmental pattern. In particular, 7- and 9-year-olds and adults in the related condition placed the objects significantly closer to the spatial category centers than they actually were. The same pattern was not observed for 11-year-olds in the related condition, however. Although they tended to place the objects closer to the spatial category centers than they actually were, the magnitude of this effect was not significant. These findings replicate the $\mathrm{U}$-shaped developmental pattern evident in our previous studies in which children and adults replaced the objects either immediately following learning or following a 12-min intervening delay (Hund \& Plumert, 2002). (A detailed discussion of possible explanations for this pattern of findings is included in the General Discussion.)

Table 2

Adjusted Ratio of Clustering (ARC) Scores for Children and Adults in Each Condition in Experiment 1

\begin{tabular}{ccc}
\hline & \multicolumn{3}{c}{ ARC score } \\
\cline { 2 - 3 } Age group and condition & $M$ & $S D$ \\
\hline 7-year-olds & & \\
Unrelated & .23 & .18 \\
Related & .74 & .29 \\
9-year-olds & & .30 \\
Unrelated & .41 & .38 \\
Related & .65 & .29 \\
11-year-olds & & .22 \\
Unrelated & .47 & .29 \\
Related & .83 & .13 \\
Adults & & \\
Unrelated & .58 & \\
Related & .94 & \\
\hline
\end{tabular}


Why did the 11-year-olds in the related condition show only minimal categorical bias? One possibility is that the cue defining the spatial categories was not salient enough for the 11-year-olds. To test this possibility, we conducted a second experiment to examine whether 11-year-olds would show categorical bias when two cues were present (i.e., object relatedness and visible boundaries). Thus, one of our goals in Experiment 2 was to investigate whether increasing the salience of the spatial category information (i.e., providing coincident categorical cues) would result in more categorical bias, especially for the 11-year-olds. A second goal was to determine whether the $U$-shaped developmental pattern revealed in Experiment 1 would be replicated.

All aspects of Experiment 2 were the same as those in Experiment 1 except that visible boundaries divided the house into four quadrants during learning. We expected that participants in the related condition would place the objects belonging to the same group closer together than would participants in the unrelated condition, which would indicate that the presence of two coincident cues (i.e., visible boundaries and object relatedness) highlights the categorical information more than does the presence of one cue (i.e., visible boundaries).

\section{Experiment 2}

\section{Method}

\section{Participants}

Ninety-six 7-, 9-, and 11-year-old children and adults participated. There were 24 participants in each age group, with approximately equal numbers of males and females in each group. The mean ages of the groups were 7 years 10 months (range $=7$ years 9 months to 8 years 1 month), 9 years 6 months (range $=9$ years 3 months to 9 years 11 months), 11 years 2 months (range $=10$ years 9 months to 12 years 5 months), and 19 years 6 months (range $=18$ years 3 months to 22 years 1 month). One additional 7-year-old and 1 additional 11-year-old were excluded because they did not reach our learning criterion. One additional adult was excluded because of experimenter error. Children and adults were recruited in the same manner as in Experiment 1.

\section{Apparatus and Materials}

The same model house, locations, and miniature objects used in Experiment 1 were used in the present experiment (see Figure 1). White lines on the floor of the house divided it into four identical quadrants $(16 \mathrm{in} . \times 16$ in. $[40.64 \mathrm{~cm} \times 40.64 \mathrm{~cm}])$ during the learning phase. The lines were $1 / 4$ in. $(0.64 \mathrm{~cm})$ tall and $5 / 16$ in. $(0.79 \mathrm{~cm})$ wide.

\section{Design and Procedure}

As in Experiment 1, participants were randomly assigned to one of two experimental conditions: related or unrelated. In the related condition, the pairings of objects and locations were constrained so that all objects belonging to one object category occupied locations in the same quadrant of the model house. In the unrelated condition, the pairings of objects and locations were completely randomized.

The learning and test phases were identical to those used in Experiment 1. The mean numbers of trials to reach the learning criterion for 7-, 9-, and 11 -year-olds and adults were $3.54(S D=1.32), 3.00(S D=1.38), 3.08$ $(S D=1.10)$, and $2.08(S D=1.14)$, respectively.

\section{Coding and Measures}

The coding of locations was identical to that in Experiment 1 . We substituted $3.33 \%$ of the locations for 7-year-olds (16 out of 480 ), $1.67 \%$ for 9 -year-olds ( 8 out of 480 ), $0 \%$ for 11-year-olds ( 0 out of 480 ), and $0.21 \%$ for adults ( 1 out of 480 ). These substituted locations were used in all analyses. We omitted $0 \%$ of locations for 7 -year-olds ( 0 out of 480 ), $0.63 \%$ for 9 -year-olds ( 3 out of 480 ), $0 \%$ for 11 -year-olds ( 0 out of 480 ), and $0.21 \%$ for adults ( 1 out of 480 ). Omitted locations were excluded from analyses.

We calculated intercoder reliability estimates of which object was placed at each of the 20 locations for 16 randomly selected participants (15\% of the sample) using exact percentage agreement. Coders agreed on $100 \%$ of the 320 locations coded. As in Experiment 1, participants received mean error, variable error, center displacement, and spatial clustering scores.

\section{Results}

\section{Mean Error}

As in Experiment 1, children and adults placed the objects fairly accurately, which suggests that they used fine-grained metric information to estimate the locations. Mean error scores were again entered into an Age (4) $\times$ Condition (2) ANOVA. This yielded a significant main effect of age, $F(3,88)=6.45, p<.01$, and a significant Age $\times$ Condition interaction, $F(3,88)=2.90, p<.05$. Simple effects tests indicated that mean error differed significantly across age groups in the unrelated condition, $F(3,44)=9.44, p<$ .01 , but not in the related condition, $F(3,44)=1.91, n s$. Fisher's PLSD follow-up tests revealed that 7-year-olds in the unrelated condition exhibited significantly greater mean errors than did 9-year-olds, 11-year-olds, and adults in the unrelated condition. No other differences were significant. Mean error scores for each age group and condition can be seen in Table 3. In summary, there were no age differences in mean error when the objects in each quadrant were related, but the 7-year-olds exhibited significantly greater errors than did the other age groups when the objects in each quadrant were unrelated.

\section{Variable Error}

To investigate possible differences in the variability of placements among the age groups and experimental conditions, we

Table 3

Mean and Variable Error Scores (in Centimeters) for Children and Adults in Each Condition in Experiment 2

\begin{tabular}{cll}
\hline Age group and condition & Mean error & Variable error \\
\hline 7-year-olds & & \\
Unrelated & $6.10(1.02)$ & $3.12(.56)$ \\
Related & $5.31(.86)$ & $2.61(.56)$ \\
9-year-olds & $4.72(1.12)$ & $2.51(.89)$ \\
Unrelated & $5.74(1.68)$ & $3.05(1.14)$ \\
Related & & \\
11-year-olds & $4.70(.94)$ & $2.21(.66)$ \\
Unrelated & $4.83(.84)$ & $2.59(.51)$ \\
Related & & \\
Adults & $4.11(.64)$ & $2.29(.46)$ \\
Unrelated & $4.65(1.35)$ & $2.13(.51)$ \\
Related &
\end{tabular}

Note. Standard deviations are listed in parentheses. 
entered variable error scores into an Age (4) $\times$ Condition (2) ANOVA. This yielded a significant main effect of age, $F(3,88)=$ 4.74, $p<.01$. The analysis also yielded a significant Age $\times$ Condition interaction, $F(3,88)=2.78, p<.05$. Simple effects tests indicated that variability decreased significantly across development in the unrelated condition, $F(3,44)=4.71, p<.01$, and in the related condition, $F(3,44)=2.99, p<.05$. In the unrelated condition, 7-year-olds' placements were significantly more variable than were the other age groups' placements. In the related condition, adults' placements were significantly less variable than were the 9-year-olds' placements. These findings show that, as in Experiment 1, variability generally decreased across development, which suggests that the precision of fine-grained information coding increased across development. Variable error scores for each age group and condition can be seen in Table 3.

\section{Center Displacement}

As in Experiment 1, we conducted two sets of analyses to examine differences in center displacement scores. In the first analysis, we entered center displacement scores into an Age (4) $\times$ Condition (2) ANOVA to explore differences in the magnitude of categorical bias across ages and conditions. This analysis yielded a significant Age $\times$ Condition interaction, $F(3,88)=2.80, p<$ .05. Simple effects tests indicated that center displacement scores were significantly greater in the related condition than in the unrelated condition for the 11-year-olds, $F(1,22)=7.97, p<.01$, and for the adults, $F(1,22)=5.74, p<.05$, but not for the other age groups, all $F_{\mathrm{s}}(1,22)<1, n s$.

In the second analysis, we conducted separate one-sample $t$ tests for each age group and condition to determine whether center displacement scores differed significantly from the expected score of 0 . Nine- and 11-year-olds and adults in the related condition and 9-year-olds and adults in the unrelated condition placed the objects significantly closer to the category centers than they actually were, all $t \mathrm{~s}(11)>2.30, p \mathrm{~s}<.05$. Seven-year-olds in the unrelated condition also placed the objects closer to the category centers than they really were, though this effect did not reach traditional significance levels, $t(11)=2.03, p<.07$. Displacement scores for 7 -year-olds in the related condition and 11-year-olds in the unrelated condition did not reach significance, all $t \mathrm{~s}(11)<1.33$, ns. Center displacement scores can be seen in Figure 3.

We also calculated a center displacement score for each individual location to determine whether the overall displacement findings were driven by only a few locations. Center displacement scores for each location were compared with the expected score of 0 by using separate one-sample $t$ tests collapsed across age groups and conditions. These analyses revealed that participants significantly displaced 13 of the locations (e.g., Locations 2, 5, 7, 8, 10, $11,12,13,14,15,16,18$, and 19) toward the centers of the groups, all $t \mathrm{~s}(95)>2.44, p \mathrm{~s}<.05$. In addition, they displaced 2 of the locations (e.g., Locations 1 and 17) away from the centers of the groups, all $t \mathrm{~s}(95)<-5.38, p \mathrm{~s}<.01$. The remaining 5 locations were not significantly displaced toward or away from the centers of the groups. Like Experiment 1, these results suggest that the overall displacement findings resulted from children and adults placing many of the locations closer to the centers of the groups than they really were. It is important to note that both inner locations (i.e., near the middle of the box) and outer locations (i.e.,

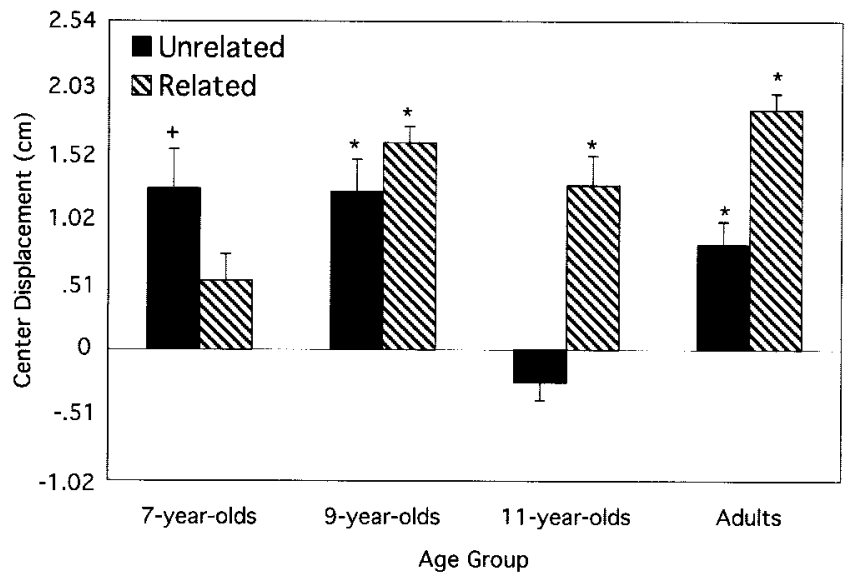

Figure 3. Center displacement scores for each age group and condition in Experiment 2. Asterisks denote significant results $(p<.05)$ of one-sample $t$ tests $(d f=11)$ comparing the displacement score with the expected score with no displacement (i.e., $0 \mathrm{~cm}$ ). The plus sign denotes marginally significant results $(p<.07)$.

near the exterior walls of the box) were displaced toward the centers of the groups. Thus, the effects were not solely based on the inner locations being pushed away from each other.

\section{Spatial Clustering}

To investigate whether the order in which participants placed the objects differed by age or condition, we entered spatial clustering (ARC) scores into an Age (4) $\times$ Condition (2) ANOVA. This analysis yielded significant main effects of age, $F(3,88)=$ $2.87, p<.05$, and of condition, $F(1,88)=31.87, p<.01$. Fisher's PLSD follow-up tests indicated that the adults exhibited greater spatial organization during test $(M=.76, S D=.24)$ than did the 7- and 11-year-olds (7-year-olds, $M=.54, S D=.33$; 9-year-olds, $M=.68, S D=.34 ; 11$-year-olds, $M=.55, S D=.40)$. As in Experiment 1, participants in the related condition were more likely to organize their replacement order by quadrant $(M=.81$, $S D=.26)$ than were participants in the unrelated condition $(M=$ $.46, S D=.36$ ). The ARC scores for each age group and condition can be seen in Table 4 .

\section{Discussion}

Our primary goal in Experiment 2 was to examine how visible boundaries and object relatedness influence children's memory for location. We were particularly interested in whether 11-year-olds would remember related objects as closer together than they really were when the groups of objects were separated by visible boundaries during learning. Eleven-year-olds in the related condition exhibited greater center displacement than did 11-year-olds in the unrelated condition. In addition, 11-year-olds in the related condition placed the objects significantly closer to the spatial category centers than they actually were. Likewise, 9-year-olds and adults in the related condition placed the objects significantly closer to the spatial category centers than they really were. Thus, when related groups of objects were separated by boundaries, children and adults exhibited categorical bias, which suggests that they 
Table 4

Adjusted Ratio of Clustering (ARC) Scores for Children and Adults in Each Condition in Experiment 2

\begin{tabular}{ccc}
\hline & \multicolumn{3}{c}{ ARC score } \\
\cline { 2 - 3 } Age group and condition & $M$ & $S D$ \\
\hline 7-year-olds & & \\
Unrelated & .48 & .35 \\
Related & .61 & .30 \\
9-year-olds & & \\
Unrelated & .48 & .35 \\
Related & .88 & .18 \\
11-year-olds & & .38 \\
Unrelated & .33 & .30 \\
Related & .77 & .38 \\
Adults & & \\
Unrelated & .55 & \\
Related & .97 & \\
& & \\
\hline
\end{tabular}

weighted categorical information heavily in their estimates of location.

Nine-year-olds and adults in the unrelated condition also placed the objects significantly closer to the category centers than they actually were, which suggests that the visible boundaries highlighted spatial category information even when the groups of objects separated by boundaries were not related. Similarly, 7 -year-olds in the unrelated condition placed the objects closer to the category centers than they actually were, though the magnitude of this effect was only marginally significant. Eleven-year-olds, however, did not place the objects closer together than they really were. Thus, when unrelated groups of objects were separated by boundaries, the magnitude of categorical bias followed a $U$-shaped developmental pattern similar to that seen when related objects were not separated by visible boundaries (see also Hund \& Plumert, 2002). In other words, when the groups of objects were either related or separated by visible boundaries, younger children and adults weighted spatial category information heavily in their estimates of location. Eleven-year-olds, however, exhibited very little categorical bias in their estimates of locations.

One exception to this overall pattern of results were the 7-yearolds in the related condition. Unlike the 7-year-olds in Experiment 1 , they did not place the objects significantly closer to the spatial category centers. Inspection of the data from individual children in both experiments revealed that this difference might have resulted from the responses of 3 seven-year-olds in the related condition in Experiment 2 who placed four or more objects within 2 in. (5.08 $\mathrm{cm}$ ) of the exterior walls of the house. Analyses of center displacement scores after exclusion of these 3 participants revealed that the remaining 7-year-olds in the related condition in Experiment 2 placed the objects significantly closer to the spatial category centers than they actually were, $t(8)=2.72, p<.05$. On average, these remaining participants placed the objects 0.40 in. $(1.02 \mathrm{~cm}$; $S D=0.45$ in. $[1.14 \mathrm{~cm}])$ closer to the spatial category centers than they really were. These findings suggest that the performance of many 7-year-olds was similar across experiments, though the performance of 3 seven-year-olds in Experiment 2 was noticeably different, which led to differences in the overall analyses of center displacement. Thus, overall, the present findings generally replicated the U-shaped pattern revealed in Experiment 1.

\section{General Discussion}

The results of this investigation clearly show that children and adults remember related objects as closer together than they really are. In Experiment 1, 7- and 9-year-olds and adults exhibited significant displacement of objects toward the centers of the spatial groups in the related condition but not in the unrelated condition. In Experiment 2, all age groups exhibited significant displacement of objects toward the centers of the spatial groups when the objects within each group were related and the groups were separated by visible boundaries. In addition, participants in both experiments were more likely to place the objects belonging to one group before moving on to another group when the objects were related than when they were unrelated. Together, these findings support the notion that information about what objects are influences memory for where objects are located.

How might object information influence location estimation? As noted previously, one possibility is that object information serves as a cue for defining spatial categories by highlighting which locations go together. These spatial categories then can be used during location estimation, thereby leading people to place items belonging to the same category closer to the center of the category than they really are. A second possibility is that associations among objects affect estimates of location through a direct crossover between the what and where systems. In other words, strong associations among objects might "leak" into memory for where the objects are located, leading people to place the objects closer together than they really are. On the basis of this view, we would expect the magnitude of categorical bias to depend on the strength of associations among the objects. Specifically, increasing the strength of the associations among the objects should lead to increases in the magnitude of categorical bias. One way to test this possibility is to vary the strength of associations among objects and observe the resulting pattern of categorical bias in estimates of location.

It is interesting that the results of the present investigation revealed U-shaped developmental patterns in the magnitude of categorical bias similar to those observed in our previous work (Hund \& Plumert, 2002). In Experiment 1, the 7- and 9-year-olds and the adults, but not the 11-year-olds, exhibited significant categorical bias in the related condition. In Experiment 2, the 7and 9-year-olds and the adults, but not the 11-year-olds, exhibited significant categorical bias when boundaries divided unrelated objects into groups. These findings have important implications for understanding how fine-grained information and categorical information are combined to produce estimates of location. As mentioned previously, the CA model proposed by Huttenlocher et al. (1991) assumes that the weighting of categorical information depends on the certainty of fine-grained metric information. When metric certainty is low, categorical information receives a higher weight, which results in distortions toward category centers. This model thus would suggest that the 11-year-olds exhibited little categorical bias because they were relatively certain about finegrained information, whereas the 7-year-olds and the adults exhibited large distortions toward category centers because they were less certain about fine-grained location information.

Although this explanation can account for why categorical bias decreased across childhood, it does not explain why adults exhibited large distortions toward category centers. The CA model 
suggests that these large biases resulted from uncertainty regarding fine-grained information. Thus, to account for the observed U-shaped pattern of results and others like it (see also Hund \& Plumert, 2002), the CA model would assume that the precision of metric coding decreases between age 11 and adulthood. However, adults placed the objects significantly closer to the true locations than did children, which suggests that adults' memory for finegrained information was very accurate. Moreover, this idea does not fit with general ideas regarding changes in memory abilities across development. Thus, the CA model's dependent weighting of categorical information does not account fully for the data obtained in the present investigation.

Instead, the present results are consistent with the independent weighting framework proposed by Hund and Plumert (2002). According to this model, estimates of location depend on the weights given to fine-grained information and categorical information at learning and on the rate of decay of fine-grained information and categorical information over time. How might we use this model to explain the results of this investigation? In the related condition in Experiment 1, 11-year-olds and adults may have encoded fine-grained metric information quite similarly, but adults may have attended more closely to categorical information than did the 11-year-olds. As a result, adults exhibited greater categorical bias than did the 11-year-olds. Likewise, although 7-, 9-, and 11-year-olds may have attended to the categorical information quite similarly, the younger children may have encoded the metric information much less precisely than did the 11-year-olds. As a result, the categorical information was weighted more heavily in the younger children's estimates of location than in the 11-yearolds' estimates. In the related condition in Experiment 2, children and adults experienced locations that were organized both by visible boundaries and object relatedness. As a result, both children and adults may have attended closely to the spatial groupings. This possibility is consistent with other research showing that younger children are more likely to exhibit categorical bias in their estimates of location when two cues highlight the categorical information (i.e., objects within a group are experienced close together in time and the groups are separated by visible boundaries during learning) than when one cue highlights category information (Hund et al., 2002; Plumert \& Hund, 2001). Further research, however, is needed to explore the factors that influence the weighting of fine-grained information and categorical information and the processes involved in combining fine-grained information and categorical information to produce estimates of location.

Why might the weighting of fine-grained information and categorical information change over development? As noted previously, our results suggest that there are developmental improvements in the accuracy and precision of coding fine-grained information. Although the mechanisms underlying this change are not well specified, the present results are consistent with previous findings suggesting that the accuracy of fine-grained coding improves throughout childhood (e.g., Acredolo \& Boulter, 1984; Cohen, Weatherford, Lomenick, \& Koeller, 1979; Hund \& Plumert, 2002; Hund et al., 2002; Plumert \& Hund, 2001; Sandberg et al., 1996; Siegel, Herman, Allen, \& Kirasic, 1979).

Developmental changes in the weighting of categorical information might result from changes in how children and adults use spatial categories to facilitate memory. Research suggests that spatial clustering is a strategy that even young children are capable of using but one that undergoes considerable change with development (e.g., Cornell \& Heth, 1986; Plumert, 1994; Plumert, Pick, Marks, Kintsch, \& Wegesin, 1994; Plumert \& Strahan, 1997; Wellman, Somerville, Revelle, Haake, \& Sophian, 1984). A major part of this development includes applying spatial clustering strategies to a broader range of tasks. One of the first ways in which children use spatial clustering is in searching for objects. Thus, 4-year-olds retrieve the objects from one cluster of locations before retrieving those in another cluster (Wellman et al., 1984). Somewhat later, children begin to use their spatial clustering skills in verbal tasks such as giving directions for finding missing objects (Plumert et al., 1994). By 12 years of age, children also use spatial clustering to structure their free recall of object locations. Thus, when asked to recall the names and locations of a set of objects, 12-year-olds recall the locations by spatial region (Plumert, 1994, Experiment 2). Finally, at around 16 years of age, adolescents apply spatial clustering to structure their recall of object names. When recalling the furniture from their homes, for example, 16year-olds, but not younger children, group furniture items by room (Plumert, 1994, Experiment 1). Together, these findings suggest that an increase in reliance on spatial categories across childhood and adolescence may underlie developmental differences in categorical weighting during location estimation.

In closing, this investigation is one of a small but growing number of studies showing that nonspatial information and spatial information interact in unexpected and nontrivial ways (e.g., Carlson-Radvansky et al., 1999; Carlson-Radvansky \& Tang, 2000; Hirtle \& Kallman, 1988; Hirtle \& Mascolo, 1986; Uttal et al., 2001). Traditionally, researchers have assumed that the processing of object information does not influence the processing of location information. This perspective is largely based on neurophysiological evidence showing that information about objects (i.e., color, shape, texture) and information about locations are processed by separate neural systems (e.g., Ungerleider \& Mishkin, 1982). Findings from the present investigation and others like it, however, suggest that information about the objects occupying particular locations influences how those locations are processed and remembered. Additional research is needed to explore how children and adults integrate these two sources of information when remembering object locations.

\section{References}

Acredolo, L. P., \& Boulter, L. T. (1984). Effects of hierarchical organization on children's judgments of distance and direction. Journal of Experimental Child Psychology, 37, 409-425.

Acredolo, L. P., \& Evans, D. (1980). Developmental changes in the effects of landmarks on infant spatial behavior. Developmental Psychology, 16, 312-318.

Carlson-Radvansky, L. A., Covey, E. S., \& Lattanzi, K. M. (1999). "What" effects on "where": Functional influences on spatial relations. Psychological Science, 10, 516-521.

Carlson-Radvansky, L. A., \& Tang, Z. (2000). Functional influences on orienting a reference frame. Memory \& Cognition, 28, 812-820.

Clayton, K., \& Habibi, A. (1991). Contribution of temporal contiguity to the spatial priming effect. Journal of Experimental Psychology: Learning, Memory, and Cognition, 17, 263-271.

Cohen, R., Weatherford, D. L., Lomenick, T., \& Koeller, K. (1979). Development of spatial representations: Role of task demands and familiarity with the environment. Child Development, 50, 1257-1260. 
Cornell, E. H., \& Heth, C. D. (1986). The spatial organization of hiding and recovery of objects by children. Child Development, 57, 603-615.

Curiel, J. M., \& Radvansky, G. A. (1998). Mental organization of maps. Journal of Experimental Psychology: Learning, Memory, and Cognition, 24, 202-214.

Engebretson, P. H., \& Huttenlocher, J. (1996). Bias in spatial location due to categorization: Comment on Tversky and Schiano. Journal of Experimental Psychology: General, 125, 96-108.

Hirtle, S. C., \& Kallman, H. J. (1988). Memory for the locations of pictures: Evidence for hierarchical clustering. American Journal of Psychology, 101, 159-170.

Hirtle, S. C., \& Mascolo, M. F. (1986). Effect of semantic clustering on the memory of spatial locations. Journal of Experimental Psychology: Learning, Memory, and Cognition, 12, 182-189.

Holyoak, K. J., \& Mah, W. A. (1982). Cognitive reference points in judgments of symbolic magnitude. Cognitive Psychology, 14, 328-352.

Hund, A. M., \& Plumert, J. M. (2002). Delay-induced bias in children's memory for location. Child Development, 73, 829-840.

Hund, A. M., Plumert, J. M., \& Benney, C. J. (2002). Experiencing nearby locations together in time: The role of spatiotemporal contiguity in children's memory for location. Journal of Experimental Child Psychology, 82, 200-225.

Hund, A. M., \& Spencer, J. P. (2003). Developmental changes in the relative weighting of geometric and experience-dependent location cues. Journal of Cognition and Development, 4, 3-38.

Huttenlocher, J., Hedges, L. V., \& Duncan, S. (1991). Categories and particulars: Prototype effects in estimating spatial location. Psychological Review, 98, 352-376.

Huttenlocher, J., Newcombe, N., \& Sandberg, E. H. (1994). The coding of spatial location in young children. Cognitive Psychology, 27, 115-147.

Kosslyn, S. M., Pick, H. L., Jr., \& Fariello, G. R. (1974). Cognitive maps in children and men. Child Development, 45, 707-716.

Mandler, J. M., \& Bauer, P. J. (1988). The cradle of categorization: Is the basic level basic? Cognitive Development, 3, 247-264

Mandler, J. M., Fivush, R., \& Reznick, J. S. (1987). The development of contextual categories. Cognitive Development, 2, 339-354.

McNamara, T. P. (1986). Mental representation of spatial relations. Cognitive Psychology, 18, 87-121.

McNamara, T. P., \& Diwadkar, V. A. (1997). Symmetry and asymmetry of human spatial memory. Cognitive Psychology, 34, 160-190.

McNamara, T. P., Halpin, J. A., \& Hardy, J. K. (1992). Spatial and temporal contributions to the structure of spatial memory. Journal of Experimental Psychology: Learning, Memory, and Cognition, 18, 555564

McNamara, T. P., \& LeSueur, L. L. (1989). Mental representations of spatial and nonspatial relations. Quarterly Journal of Experimental Psychology: Human Experimental Psychology, 41A, 215-233.

Newcombe, N., \& Liben, L. S. (1982). Barrier effects in the cognitive maps of children and adults. Journal of Experimental Child Psychology, 34, $46-58$.

Plumert, J. M. (1994). Flexibility in children's use of spatial and categorical organizational strategies in recall. Developmental Psychology, 30, $738-747$.

Plumert, J. M., \& Hund, A. M. (2001). The development of memory for location: What role do spatial prototypes play? Child Development, 72 , $370-384$.

Plumert, J. M., Pick, H. L., Jr., Marks, R. A., Kintsch, A. S., \& Wegesin, D. (1994). Locating objects and communicating about locations: Organizational differences in children's searching and direction-giving. Developmental Psychology, 30, 443-453.

Plumert, J. M., \& Strahan, D. (1997). Relations between task structure and developmental changes in children's use of spatial clustering strategies. British Journal of Developmental Psychology, 15, 495-514.

Roenker, D. L., Thompson, C. P., \& Brown, S. C. (1971). Comparison of measures for the estimation of clustering in free recall. Psychological Bulletin, 76, 45-48.

Sadalla, E. K., Burroughs, W. J., \& Staplin, L. J. (1980). Reference points in spatial cognition. Journal of Experimental Psychology: Human Learning and Memory, 6, 516-528.

Sandberg, E. H., Huttenlocher, J., \& Newcombe, N. (1996). The development of hierarchical representation of two-dimensional space. Child Development, 67, 721-739.

Sherman, R. C., \& Lim, K. M. (1991). Determinants of spatial priming in environmental memory. Memory \& Cognition, 19, 283-292.

Siegel, A. W., Herman, J. F., Allen, G. L., \& Kirasic, K. C. (1979). The development of cognitive maps of large- and small-scale spaces. Child Development, 50, 582-585.

Spencer, J. P., \& Hund, A. M. (2002). Prototypes and particulars: Geometric and experience-dependent spatial categories. Journal of Experimental Psychology: General, 131, 16-37.

Spencer, J. P., \& Hund, A. M. (in press). Developmental continuity in the processes that underlie spatial recall. Cognitive Psychology.

Ungerleider, L. G., \& Mishkin, M. (1982). Two cortical visual systems. In D. J. Ingle, M. A. Goodale, \& R. J. W. Mansfield (Eds.), Analysis of visual behavior (pp. 549-586). Cambridge, MA: MIT Press.

Uttal, D. H., Gregg, V. H., Tan, L. S., Chamberlin, M. H., \& Sines, A (2001). Connecting the dots: Children's use of a systematic figure to facilitate mapping and search. Developmental Psychology, 37, 338-350.

Wellman, H. M., Somerville, S. C., Revelle, G. L., Haake, R. J., \& Sophian, C. (1984). The development of comprehensive search skills. Child Development, 55, 427-481.

Received August 28, 2002

Revision received April 9, 2003

Accepted May 1, 2003

\section{Wanted: Your Old Issues!}

As APA continues its efforts to digitize journal issues for the PsycARTICLES database, we are finding that older issues are increasingly unavailable in our inventory. We are turning to our long-time subscribers for assistance. If you would like to donate any back issues toward this effort (preceding 1982), please get in touch with us at journals@apa.org and specify the journal titles, volumes, and issue numbers that you would like us to take off your hands. 